\title{
Elementos críticos de la escuela en territorio mapuche ${ }^{1}$
}

\section{Elementos críticos da escola em território mapuche}

\section{Critical elements of the school in the mapuche territory}

\author{
Héctor Torres Cuevas* \\ Miguel Friz Carrillo*
}

\begin{abstract}
RESUMEN
El artículo presenta elementos críticos que caracterizan el funcionamiento de la escuela en territorio mapuche a partir de lo reflexionado con miembros de comunidades mapuches que cumplen roles a nivel familiar, social y político. La metodología de la investigación se sitúa en la antropología de la educación lo que ha favorecido una aproximación cualitativa del objeto de estudio. Los resultados evidencian dificultades de acceso a la escuela, cuestionamientos al perfil de los proyectos educativos, el establecimiento de relaciones de poder asimétricas familia-escuela y el predominio de una educación desterritorializada. Se concluye que estos elementos tensionan la construcción de procesos políticos destinados a favorecer la libre determinación educativa de la sociedad mapuche.
\end{abstract}

Palabras clave: Proyectos educativos. Mapuche. Colonialismo. Relación familia-escuela.

1 Este artículo agradece el financiamiento del proyecto Fondo Nacional de Desarrollo Científico y Tecnológico (FONDECYT) N 11171177 “Contextualización y territorialización de la gestión educativa de la escuela en La Araucanía” y del proyecto FONDECYT N 1180993 "Estudio sobre las configuraciones del conocimiento matemático que desarrollan profesores en comunidades educativas interculturales situadas en contextos de ruralidad, migración y comunidades mapuche".

*Universidad del Bío Bío. Chillán, Ñuble, Chile. E-mail: htorres@ubiobio.cl - https://orcid. org/0000-0001-9762-7245 E-mail: mfriz@ubiobio.cl - https://orcid.org/0000-0002-5945-9559 


\title{
RESUMO
}

$\mathrm{O}$ artigo apresenta elementos críticos que caracterizam o funcionamento da escola em território Mapuche a partir de uma reflexão com integrantes de comunidades Mapuches que cumprem funções no âmbito familiar, social e político. A metodologia da pesquisa situa-se na antropologia da educação, o que favoreceu uma abordagem qualitativa do objeto de estudo. Os resultados apontam dificuldades no acesso à escola, questionando do perfil dos projetos educacionais, o estabelecimento de relações de poder assimétricas famíliaescola e a predominância de uma educação desterritorializada. Conclui-se que esses elementos enfatizam a construção de processos políticos destinados a favorecer a livre determinação educacional da sociedade Mapuche.

Palavras-chave: Projetos educacionais. Mapuche. Colonialismo. Relacionamento família-escola.

\begin{abstract}
The article presents critical elements that characterize the functioning of the school in the Mapuche territory from the reflection along Mapuche communities' members that play familiar, social and political roles. The research's methodology is located in the education anthropology, which has worked in favor of a qualitative approximation of the study's object. The results show access difficulties to school, educational projects profile questionings, asymmetrical school-family power relationship establishments and the prevalence of a de-territorialized education. It is concluded that these elements tension the political processes creation destined to favor the free Mapuche society educational determination.

Keywords: Educational projects. Mapuche. Colonialism. Family-school relationship.
\end{abstract}

\section{Introducción}

La escuela en contextos indígenas ha operado, en una perspectiva histórica, como una institución de origen exógeno sostenida en un modelo de formación eurocéntrico, creado para colonizar a los estudiantes indígenas (BOUSQUET, 2012; SIMPSON, 2014; MANSILLA; HUAIQUIAN; POZO, 2018). De esta manera, el trabajo concretado por los actores educativos en la escuela ha propiciado transformaciones del mundo social indígena (LOPES DA SILVA, 2009) en una doble perspectiva: 1) Al concretar la asimilación lingüística y cultural de 
niños y jóvenes (WEENIE, 2008; PORMA, 2015; COMMISSION DE VÉRITÉ ET RÉCONCILIATION DU CANADA, 2015); y 2) al propiciar formas de resistencia y apropiación del espacio escolar que muestran la capacidad de agencia de los pueblos indígenas (MCCARTY, 2010; GONZÁLEZ APODACA, 2013; GARCIA; SHIRLEY, 2015; BERTELY; SARTORELLO; ARCOS, 2015; LOPES, 2016).

En este sentido, organizaciones, líderes e intelectuales indígenas han puesto en tensión el modelo de escuela colonizador, al ser incoherente con los desafíos que emergen con los procesos de construcción de libre determinación (MCCARTY, 2010). Esto ha llevado al surgimiento de diferentes respuestas como el reconocimiento estatal de marcos de autonomía para la gestión educativa de la escuela (PAQUETTE; FALLON, 2010; MCGREGOR, 2012), la implementación de programas de educación intercultural bilingüe (PEIB) en zonas con alta concentración de población indígena (LÓPEZ, 2016) y el surgimiento de escuelas y programas alternativos, construidos desde abajo por educadores y organizaciones indígenas (BERTELY; SARTORELLO; ARCOS, 2015; TOCKER, 2015; NIGH; BERTELY, 2018).

Desde esta perspectiva, situando nuestro análisis a lo que se experimenta en la región de La Araucanía (Chile), se constata la necesidad de avanzar en la discusión política sobre los marcos de referencia que debieran orientar a la escuela en territorio mapuche. En la actualidad, la existencia del PEIB ha favorecido la enseñanza de saberes mapuches en las escuelas (QUIDEL, 2011; LUNA; BENAVIDES; GUTIÉRREZ, 2014). No obstante, su impacto ha sido limitado y no ha generado transformaciones que aporten a revertir el conflicto intercultural y epistémico vigente en el medio escolar (QUILAQUEO; SARTORELLO, 2018). Por eso mismo, hemos iniciado un trabajo enfocado a dialogar con miembros de comunidades mapuches que cumplen roles a nivel familiar, social y político, con el propósito de transitar de una educación escolar que ha excluido las metas e intereses de la población indígena, a un sistema que ponga en el centro sus aspiraciones y necesidades (RICO, 2013).

El artículo presenta elementos que son considerados críticos en el funcionamiento de la escuela en territorio mapuche, desde la perspectiva de actores que participan de manera significativa a nivel local y regional, en la reivindicación de derechos sociales, culturales y políticos del pueblo Mapuche ante el Estado chileno. 


\section{Marco de referencia}

\section{Enfoque misional y colonizador de la escuela en territorio mapuche}

La escuela en territorio mapuche ${ }^{2}$ es el resultado de procesos históricos, políticos y sociales, que han forjado el carácter y funcionamiento de la institución escolar. En este sentido dos elementos fundantes de la escuela son su enfoque misional y colonizador. El enfoque misional de la escuela ha tenido como orientación conseguir la evangelización de la sociedad mapuche en los valores y creencias del cristianismo (BOCCARA; SEGUEL, 1999; SÁNCHEZ, 2011; CANDIADA SILVA, 2017). Este proceso se inició en la etapa colonial (1598-1810) con la labor de misioneros jesuitas y franciscanos, lo que fue profundizado desde el siglo XIX por el Estado chileno al incorporar nuevos actores como misioneros capuchinos y anglicanos (FLORES; AZÓCAR, 2006; SERRANO; PONCE DE LEÓN; RENGIFO, 2013; MANSILLA; LLANCAVIL; MIERES; MONTANARES, 2016; CANO, 2018). Así, las escuelas misionales se encargaron (antes que la escuela pública) de la educación de la población mapuche, en los territorios que les fueron usurpados por el ejército chileno (1860-1883).

Articulado con el enfoque misional se encuentra el rol colonizador de la escuela en el sentido de ejercer el control cultural, lingüístico, educativo y territorial de la población mapuche (MANSILLA; LLANCAVIL; MIERES; MONTANARES, 2016). Para ello, el currículum escolar se basó en un programa de estudios diseñado en la lógica de la sociedad dominante y consideró aspectos como la enseñanza del castellano, religión, aritmética, historia, geografía, dibujo y trabajos manuales (CANDIA DA SILVA, 2017; RESCHIO, 2018). De esta manera, la escuela se incorporó con el objetivo de dominar a través de un proyecto educativo, que concibió al mapuche como un sujeto que debía ser civilizado, por medio de su evangelización y sujeción a los marcos de la sociedad

2 Según la Comisión de Trabajo Autónoma Mapuche (GOBIERNO DE CHILE, 2003, p. 715). El Gulumapu, se extiende entre el río Limarí (zona norte) hasta la Isla Grande de Chiloé (zona sur), desde la Cordillera de Los Andes al mar Pacífico. El Puelmapu, se extiende entre el río IV y el río Diamante, por el Norte, hasta el río Limay por el Sur, siendo su límite Este el río Salado en las cercanías de Buenos Aires y el Oeste la Cordillera de Los Andes. En la actualidad la sociedad mapuche se encuentra divida por las fronteras definidas por los estados de Chile y Argentina. En el caso de Chile los mapuches fueron instalados en un sistema de reducciones creado por el Estado a través de la "Comisión Radicadora de Indígenas" (1884-1929), quedando bajo su control un 10\% (510.385 hectáreas) de su territorio ancestral. 
nacional chilena (PINTO, 2003; PORMA, 2015; CANO, 2018). Así, según lo documentado por Mansilla Huaiquian y Pozo (2018), se favorecieron procesos de disciplinamiento de la sociedad mapuche, en la lógica de generar un orden social coherente con los discursos y prácticas de una sociedad racista, colonial y cristiana. En este contexto, la escuela que se instala en territorio mapuche mantendrá vigente durante todo el siglo XX su enfoque misional y colonizador, lo que comenzará a ser discutido de manera formal con la implementación del PEIB en la década de $1990^{3}$.

\section{Transformación neoliberal del sistema escolar chileno}

Un elemento de orden contemporáneo que ha transformado el funcionamiento de la escuela en territorio mapuche, es la instauración en el sistema escolar chileno de un cuasi-mercado escolar en la década de 1980 (ELACQUA; SANTOS, 2013; VILLALOBOS; QUARESMA, 2015). La neoliberalización de la educación chilena en la década de 1980, significó poner fin al sistema nacional de educación pública, transfiriendo el Estado la gestión de las escuelas públicas a los municipios y alentándose la creación de instituciones educativas privadas con subvención estatal (ALMONACID, 2004; SLACHEVSKY, 2015).

De esta manera, el Estado asumió que la descentralización en la gestión de las escuelas públicas, la libertad de elección de los padres, la introducción de un sistema de vouchers y la competencia entre escuelas provocaría una mejora en la calidad de la educación (VILLALOBOS; QUARESMA, 2015). Lo anterior es algo que no ha sido logrado, destacándose el sistema escolar chileno por su alto nivel de segregación, el desequilibrio en los resultados escolares entre instituciones públicas y privadas y el debilitamiento estructural de la educación pública (BELLEI, 2007; VALENZUELA; BELLEI; DE LOS RÍOS, 2010; VILLALOBOS; VALENZUELA, 2012; DONOSO; CASTRO;

3 Es importante destacar que existen antecedentes históricos previos que muestran la preocupación de la sociedad mapuche de discutir el rol de la escuela en sus territorios. Por ejemplo, es lo que hicieron durante las primeras décadas del siglo XX la Junta General de Caciques del Futawillimapu y docentes mapuches como Zoila Quintremil, Zenobia Quintremil e Ignacio Huenchullan. De esta forma antes de la formulación de la política del PEIB ya se había exigido al Estado la mejora en la cobertura escolar, escuelas que consideraran el carácter rural de la vida de la población mapuche, la formación e incorporación de docentes mapuches a cargo de las escuelas y la enseñanza en mapuzugun y español. Si bien, lo exigido no fue atendido por el Estado chileno constituyó un precedente histórico para lo que se inicia a partir de la década de 1990. 
ALARCÓN, 2015). Estos elementos críticos han llevado a la materialización de una reforma educativa durante el segundo gobierno de Michelle Bachelet (2014-2018), destacándose entre las medidas el proceso de desmunicipalización de establecimientos educativos, los que han comenzado a ser traspasados a una nueva estructura denominada Servicios Locales de Educación.

Bajo este escenario, en territorios mapuche de La Araucanía la oferta educativa está compuesta por tres tipos de instituciones: escuelas municipales, escuelas particulares subvencionada (sin copago por parte de la familia) y escuelas pertenecientes al Servicio Local de Educación Costa Araucanía ${ }^{4}$. Si bien hay proyectos educativos que intentan responder de manera particular a las características sociales, culturales y territoriales de las comunidades mapuches (LUNA; BOLOMEY; CANIGUAN, 2018), en la práctica no existe un sistema de escuelas que se encuentre bajo gobernanza y control de organismos o miembros de las comunidades mapuches. Entonces, las familias mapuches disponen de un sistema de escuelas que se caracteriza por poseer proyectos educativos descontextualizados, que priorizan el cumplimiento del marco curricular del Ministerio de Educación, la evaluación estandarizada del Sistema de Medición de la Calidad de la Educación (SIMCE) y la competencia por matrícula escolar (TORRES, 2017).

\section{Política multicultural chilena: Programa de Educación Intercultural Bilingüe (PEIB)}

La política multicultural del Estado chileno ha incorporado, desde fines de la década de 1990, el PEIB como una forma de responder a las exigencias de los pueblos indígenas de contar con una educación escolar que respete y valore su identidad, lengua y cultura (FUENZALIDA, 2014; CASTILLO; LONCÓN, 2015). De esta manera, el PEIB ha intentado revertir la falta de pertinencia de los proyectos educativos en contextos escolares indígenas a través de medidas como las siguientes:

a) Focalización de escuelas para interculturalizar el proyecto educativo;

b) Entrega de asistencia técnica a las escuelas focalizadas;

c) Elaboración de material didáctico (textos de estudio);

d) Introducción en el programa de estudios de la asignatura de Lengua Indígena;

4 El Servicio Local de Educación Costa Araucanía ha comenzado a funcionar el año 2018 y agrupa las escuelas públicas de 5 municipios: Carahue, Nueva Imperial, Saavedra, Teodoro Schmidt y Toltén. 
e) Incorporación de educadores tradicionales para la enseñanza de la lengua y cultura indígena (MINISTERIO DE EDUCACIÓN [MINEDUC], 2005a, 2005b, 2017; FUENZALIDA, 2014).

Cada una de estas medidas ha favorecido una apertura intercultural que no había sido considerada en las escuelas en territorio mapuche (WILLIAMSON; FLORES, 2015). Es así que hoy es posible constatar la incorporación de contenidos y estrategias, que favorecen la enseñanza de la lengua y cultura mapuche en los niveles de educación primaria (QUIDEL, 2011; LUNA; BENAVIDES; GUTIÉRREZ, 2014; SOTOMAYOR; ALLENDE; FUENZALIDA; HASLER; CASTILLO, 2015; CALDERÓN; CASTILLO; FUENZALIDA; HASLER; MARIANO; VARGAS, 2017).

Sin embargo, un análisis crítico de las medidas movilizadas por el PEIB nos muestra que los esfuerzos han sido insuficientes para transformar el eurocentrismo de los contenidos escolares y la hegemonía del español como primera lengua de enseñanza (POZO, 2014; LAGOS, 2015). Menos aún han sido un aporte a la construcción de marcos políticos de participación, a nivel familiar y comunitario, que tengan por objeto la libre determinación educativa. Siguiendo el análisis de Tubino (2011), los programas de educación intercultural bilingüe que hemos visto hasta ahora en América Latina, más que apuntar a una ruptura con el estatus quo o la exclusión social de los indígenas, representan eslóganes de propaganda funcionales al sistema económico neoliberal, que suenan muy progresistas cuando se habla públicamente de interculturalidad para todos, pero que en la práctica quedan destinados exclusivamente a la población indígena. Es así como el Estado neoliberal ha encontrado en la política multicultural, la respuesta ideológica para orientar su relación contemporánea con los pueblos indígenas, al igual como el indigenismo fue utilizado por los Estados populistas para la integración y asimilación de los indígenas durante la segunda mitad del siglo XX (BONFIL BATALLA, 1992; FAVRE, 1996; BOLADOS, 2012). Esto es significativo si se piensa que el proceso de apertura democrática e inclusión que vivimos en Chile, es una readaptación del sistema de control de la diferencia, bajo parámetros que se muestran respetuosos frente a la alteridad, que la reconocen, pero que no han significado un cambio en las condiciones asimétricas de relación entre el Estado y los Pueblos Indígenas (BOCCARA; AYALA, 2011; BRIONES, 2014). 


\section{Metodología}

La investigación se sitúa en un enfoque antropológico de la educación, con el propósito de realizar la documentación de conocimientos sociales, culturales y políticos mapuches, que permitan discutir los elementos críticos de la gestión educativa de la escuela en La Araucanía-Chile. De manera sintética los elementos que han caracterizado el trabajo antropológico son los siguientes:

a) Enfocarse en las situaciones que se viven cotidianamente, lo que no aparece en el formato de lo público, lo informal, lo obvio, pero también los intereses que se tejen por quienes tienen poder y que no aparece por escrito;

b) Incorporación en el trabajo etnográfico de una perspectiva histórica;

c) Se concibe al sujeto como un activo constructor del mundo social (capacidad de agencia y resistencia);

d) Análisis de las formas apropiación que ponen en práctica los sujetos;

e) Presencia permanente del etnógrafo en el terreno y su involucramiento personal y subjetivo para documentar lo no documentado (Rockwell, 1997, 2009). El trabajo de terreno se realizó con miembros de comunidades mapuches que residen en 5 municipios (comunas) de la región de La Araucanía: Ercilla, Galvarino, Chol-Chol, Temuco y Padres las Casas. La región de La Araucanía se ubica en la zona centro-sur de Chile, forma parte del territorio históricamente habitado por el pueblo Mapuche (DILLEHAY; SAAVEDRA, 2010) y en la actualidad está dividida político-administrativamente en 32 municipios. Según datos del censo 2017 de las 957.224 personas que viven en la región un $34,3 \%$ de la población declara pertenecer a un pueblo indígena, predominando la población mapuche (más de 300 mil personas).

En esta etapa de la investigación trabajamos con 14 miembros de comunidades mapuches, los que han sido contactados por tener al interior de sus comunidades responsabilidades sociales, culturales y políticas, abarcando: autoridades tradicionales (logko, werken), representantes de su comunidad, miembros de organizaciones mapuches, militantes políticos y defensores del territorio. Para documentar la información hemos realizado entrevistas semiestructuradas, conversaciones, notas de campo y dos talleres de discusión. Los ejes temáticos discutidos con los actores políticos han sido los siguientes:

a) libre determinación educativa en contexto mapuche;

b) relación escuela-familia-comunidad;

c) contemporaneidad contexto escolar mapuche;

d) participación familiar y comunitaria;

e) liderazgo desde el conocimiento mapuche. 
En todas las etapas del trabajo, utilizamos consentimiento informado, con el propósito de garantizar una participación informada y voluntaria de cada persona que ha colaborado en la investigación. Con el propósito de resguardar el anonimato de los participantes, en la presentación de los resultados no utilizamos sus nombres.

El procesamiento de los datos se basó en el análisis de contenido con el propósito de realizar la lectura, interpretación y comprensión del contenido manifiesto y latente presente en los datos recopilados (MILES; HUBERMAN, 2003). La técnica que se utilizó nos entregó la posibilidad de realizar un procesamiento metódico y en profundidad de los datos, para generar un conjunto emergente de códigos y categorías basado en los conocimientos de los actores en el nivel familiar, escolar, comunitario y político (QUIVY; CAMPENHOUDT, 1998). De esta manera, el análisis se orientó por las siguientes etapas:

a) Preparación y organización del material de campo;

b) Inicio de la lectura del material;

c) Generación del ordenamiento conceptual de los datos (codificación);

d) Creación de memos para sistematizar comentarios sobre el proceso de análisis;

e) Identificación de categorías a partir de las características del contenido, siendo definidas desde un método que considerará elementos inductivos;

f) Revisión de la coherencia de las categorías y los códigos;

g) Aseguramiento de la calidad (viabilidad y factibilidad) de las categorías y códigos por medio de la utilización de la triangulación de datos y la saturación de contenidos;

h) Escritura del informe de análisis (L'ÉCUYER, 1990; BARIBEAU, 2009).

\section{Resultados}

Los Elementos críticos de la escuela en territorio mapuche dan cuenta de características del funcionamiento de la escuela, aspectos históricos vinculados a la escolarización de la población mapuche y el tipo de relaciones que se establece entre la familia y la escuela. Al respecto, los elementos identificados por los miembros de comunidades mapuches se refieren a:

a) Acceso a la escuela y perfil de los proyectos educativos en territorio mapuche;

b) Relación de poder asimétrica familia- escuela;

c) Escuela desterritorializada. 


\section{Acceso a la escuela y perfil de los proyectos educativos en territorio mapuche}

Un primer elemento que cuestionan los actores involucrados en la investigación tiene relación con el acceso a la escuela y el perfil de los proyectos educativos institucionales (PEI) en territorio mapuche. Al respecto, existe disconformidad por la dificultad de las familias de acceder al interior de sus comunidades a escuelas que entreguen educación desde el nivel primario al secundario. Si bien, en territorios rurales de La Araucanía funcionan 659 escuelas, la mayoría de las instituciones entrega educación hasta el nivel de educación básica (primaria). Esto obliga a los padres a buscar alternativas para la continuidad de estudio de sus hijos en los centros urbanos más próximos a sus comunidades. Esto fue señalado por una madre en el segundo foro taller, al relatar que sus dos hijas al completar sexto básico, se vieron en la obligación de continuar su educación en la ciudad de Victoria en un colegio con orientación católica (Nota de campo, 27/09/2018).

Lo anterior es la norma que se repite para el conjunto de padres que entrevistamos, siendo los principales centros urbanos receptores de sus hijos Traiguén, Galvarino, Ercilla, Padre Las Casas, Chol-Chol y Victoria.

No obstante, este problema no es exclusivo de la educación secundaria, siendo observado en el nivel primario de educación, producto del cierre de escuelas rurales (NÚÑEZ; SOLÍS; SOTO, 2014). Es así como una madre en el primer foro taller relató que para llegar a la reunión de padres y apoderados debía caminar alrededor de 16 kilómetros, ya que la escuela donde tiene a su hijo es la única opción disponible, al haber cerrado el municipio los establecimientos cercanos a su hogar por tener baja matrícula escolar (Nota de campo, 7/06/2018). Siguiendo lo analizado por Flores y Carrasco (2016), lo que sucede en territorio mapuche, es la expresión de la inequidad espacial de la oferta local de educación en el sistema escolar chileno, lo que deriva en que las familias matriculen a sus hijos en lo que tienen disponible geográficamente. Sumado a la inequidad del acceso a escuelas se cuestiona la orientación y énfasis de los PEI. En este sentido el miembro de una comunidad relató lo siguiente:

Salió hermoso el Proyecto Educativo Institucional, pero hasta el día de hoy no veo nada, nunca nos han llamado para conversar este tema, las conversaciones cuando se hacen las reuniones de apoderados son conversaciones para ver los avances: "si les fue bien en matemática, colóquele más empeño en lenguaje”. Entonces nos pasamos hablando 
de los avances que deben llevar los niños y las niñas y el SIMCE que es otro punto que se pone mucha atención, que nos preocupamos de este tema porque la escuela será considerada a nivel regional. De hecho, ya pasó a ser mejor escuela comunal y eso después de la región. Entonces hemos sentido que nuestra hija llega estresada, o sea, llega con un nivel de estrés que los presionan mucho para el SIMCE, incluso con estrategias reñidas éticamente, haciendo ofrecimientos para que los niños puedan sacar mejor rendimiento, los vienen a invitar a la nieve "los vamos a premiar por eso". Lo único que falta es que digan que nos van a dar una caja de comida, es lo único que falta (APM1 [4:4]).

En la perspectiva de lo analizado en las conversaciones, se constata que en las escuelas en territorio mapuche se materializan proyectos educativos, que tienen mayor preocupación por los resultados de las pruebas de medición como SIMCE y en la cobertura de los contenidos mínimos del plan curricular nacional. Como ha dicho un logko, todo es individualismo y competitividad en la educación que reciben los niños, se carece de proyectos que prioricen el arraigo cultural y territorial, o que coloquen en práctica procesos formativos que articulen los conocimientos curriculares con los conocimientos mapuches (Nota de campo, 27/09/2018). De esta manera, lo que existe en términos de oferta educativa es una escuela basada en valores neoliberales, que tiene entre sus principales desafíos demostrar que pueden ser instituciones capaces de ser exitosas en la obtención de resultados académicos estandarizados (WEINSTEIN; MUÑOZ; MARFÁN, 2012; ASSAÉL; ACUÑA; CONTRERAS; CORBALÁN, 2014). En este sentido, lograr la competitividad académica permite a los docentes de estas escuelas acceder a reconocimientos como la Subvención por Desempeño de Excelencia (MIZALA; ROMAGUERA, 2002). Además, garantiza a los directores de estas instituciones mostrar que son eficientes en el uso de los recursos públicos, en el marco de un sistema escolar que tiene incorporado mecanismos de rendición de cuentas basados en indicadores estandarizados (PARCERISA; FALABELLA, 2017).

\section{Relación de poder asimétrica familia-escuela}

Un segundo elemento que se discute por parte de los actores políticos es el carácter asimétrico de la relación de poder entre la familia y la escuela, lo que se expresa en prácticas y conductas que están presentes al interior de las comunidades educativas. Al respecto, el miembro de una comunidad señaló lo siguiente: 
Yo creo que todavía existen padres y apoderados mapuches que tienen ese miedo a sacar la voz, a decir sus planteamientos porque ellos anteriormente cuando fueron al colegio recibieron también discriminación y solamente lo que decía el profesor se hacía y no se podía cuestionar de ninguna manera. Es lo que dije al colegio se iba solamente a obedecer, como que no tenía capacidad de opinar [...] (APM2 [11:11]).

En los actores del medio familiar la educación autoritaria, la discriminación y la asimilación lingüística, han dejado huellas que se expresan en su forma de relacionarse con los profesores en el medio escolar (PORMA, 2015; TORRES, 2016). Es lo que explicaría para los actores políticos el silencio en las reuniones de padres y apoderados, ya que los padres mapuches no se sentirían en confianza y validados como sujetos con derecho a participar de la toma de decisiones. Es lo que nos señaló el miembro de una comunidad al decir que:

"[...] hoy día lo que hacen los viejitos es que van a la reunión y no dicen nada, porque sienten que el profesor, no tienen la apertura para recibir lo que ellos le están entregando, no hay confianza porque dice "qué dirá", "se reirá de lo que yo le diga" [...]" (APM1 [24:24]).

Esto refuerza la vigencia de relaciones que no son constructivas, ya sea por vergüenza o temor a equivocarse frente a una figura de poder, como se observa en el siguiente relato:

La última vez que fui a la reunión de padres, tenían un reclamo con la profesora de segundo, éramos dos, pero los demás agachan el moño, la mayoría son apoderados viejitos, son mayores; los abuelos al cuidado de sus nietos son súper sumisos, están todos, ellos mandan a su hijo a lo que es correcto, pero no cuestionan nada de lo que dice la profesora, ni el horario, o porque a tu hijo no lo dejan ir al baño, o sea esta profe puede mandar, no está el plano de igualdad, y de hecho la profesora es mapuche, y no se ve, ella va a dar clases nada más (APM5 [8:8]).

Así, lo que se impone es el obedecer sin cuestionar, un orden que ampara el privilegio de los profesores de tomar decisiones y de los padres de acatar lo que se dicta en el medio escolar. Sobre esto, un dirigente mapuche indicó que el problema 
es "[...] que nuestra gente es sumisa, solamente tiene la visión de participar, pero no de imponerse, no tiene interés que cambie ese colegio [...]" (APM3 [28:28]). Lo explicitado en los testimonios da cuenta de tres aspectos críticos:

a) un cuestionamiento intra-comunitario a la actitud que asumen los padres y abuelos en el medio escolar;

b) la naturalización de una forma de participación de los padres de familia que evita conflictuar el rol de la escuela en sus territorios;

c) una deficiente comunicación entre profesores y padres de familia.

De esta manera, la desigual distribución del poder entre los actores que conforman la comunidad educativa, es una expresión de la desconfianza que se tiene hacia la familia mapuche por parte de profesores y directivos, de manera similar a lo que se expresa en otros contextos indígenas (SALAÜN; VERNAUDON, 2009). En este sentido, la familia es útil cuando se ajusta de manera instrumental a lo que se exige por parte de la escuela, quedando en un segundo plano la generación de procesos democráticos en la toma de decisiones.

\section{Escuela desterritorializada}

Un tercer aspecto que es cuestionado por los actores políticos tiene relación con el hecho que la escuela es una institución que no está articulada con las necesidades, conocimientos y características que configuran el contexto territorial. Al respecto una dirigente mapuche explicó lo siguiente:

No hay un conocimiento cultural ni de ningún tipo para que se pueda dar este proceso de enseñanza del colegio hacia los niños y los apoderados, no hay eso, no se encuentra eso y eso mismo dificulta el que los profesores que vienen tratan a los niños de la misma forma que en el sector urbano, lo cual aqui por las condiciones geográficas, culturales, de idioma y un montón de cosas tendría que ser diferente, tendría que ser diferente porque claramente los niños, las familias, no están a la misma altura que un sector urbano y no tienen por qué estarlo tampoco, porque nosotros vivimos según nuestras condiciones, nuestra costumbre, nuestra cultura [...] (APM5 [022:031]). 
Lo argumentado expresa una disconformidad con los marcos de referencia que orientan el funcionamiento de la escuela, al reproducir lo que se hace en sectores urbanos no mapuches. En este sentido, se excluyen del proceso de escolarización elementos geográficos, culturales, sociales y ambientales, que caracterizan la vida de las familias mapuches en el medio comunitario. Sobre esto el miembro de una comunidad planteó:

[...] cada comunidad tiene su fuerza espiritual, aqui está la fuerza del río, el río para la escuela no significa nada, no he oído hablar nunca del río en la escuela, la importancia del río, de ir a dar una vuelta al río, es como agua que pasa nada más. Pero si uno se da cuenta antiguamente la ceremonia que se realizaba, la gente tenía mucho respeto por el rio y no solamente eso sino todo el conocimiento acerca de cómo la espiritualidad estaba asociada a la forma en que los seres humanos se relacionan unos con otros. (APM1 [12:12]).

Esto reafirma la vigencia de una distancia epistémica y el menosprecio por el conocimiento mapuche en el medio escolar, al considerarse que los niños y jóvenes deben ser escolarizados con los marcos de conocimiento de la sociedad dominante. Un dirigente mapuche analizaba que en la escuela las jornadas de clase son extensas y pareciera que todo solo fuera teoría, a lo que argumentó la necesidad de contar con procesos de enseñanza y aprendizaje vinculados con actividades prácticas en el territorio (APM2 [19:19]). Mientras tanto, otro padre de familia señaló que en su perspectiva la escuela es igual que una caja cerrada, ya que no se preocupa de ver lo que sucede en el territorio o de solucionar situaciones problemáticas recurriendo a conocimientos que están presentes en el medio familiar y comunitario. A su vez, un dirigente mapuche planteó que:

"La educación que se hace en este país, se hace para ser individualista total, por lo tanto, no viene con ningún sentido social de apoyar, ellos [los profesores] vienen a hacer su clase y listo, no miran que es lo que pasa con la sociedad, en que los podemos integrar, a quien le podemos mejorar o enseñar algo más, no ven eso" (APM8 [00.37:01.16]).

En resumen, lo que tenemos es una escuela desconectada del territorio, que desconoce los elementos que son valorados por la sociedad mapuche y también 
aspectos críticos de la vida comunitaria tales como la dificultad de acceso al agua, el impacto social y ambiental de la industria forestal, el empobrecimiento económico, la migración por trabajo y los conflictos intra-comunitarios.

\section{Discusión y conclusiones}

A modo de discusión, lo relatado por cada uno de los participantes en el estudio constituye una evidencia de la necesidad de conflictuar el sentido y las prácticas de la institución escolar, desde una conciencia indígena crítica de los procesos de dominación colonial experimentados por la sociedad mapuche. De esta manera, los marcos de referencia de la escuela en territorio mapuche están anclados a un modelo social, económico y político que refleja:

a) la vigencia del colonialismo como una forma de control de la sociedad mapuche que vive en los territorios comunitarios;

b) el predominio de una educación neoliberalizada;

c) la ausencia de procesos políticos destinados a favorecer la libre determinación educativa de la sociedad mapuche.

En este sentido, los marcos de referencia de la escuela han permitido sostener prácticas que son cuestionadas al no favorecer una transformación de las relaciones de poder en el espacio escolar. Así, la institución escolar en contextos indígenas sigue marcada por el autoritarismo y una cultura interna que evita la generación de procesos democráticos para la toma de decisiones (SALAÜN; VERNAUDON, 2009). Esto se ve reforzado por las actitudes de padres de familia que optan por el silencio, la sumisión o el desinterés, para evitar la confrontación de sus puntos de vista con lo planteado por los profesores. Al respecto, lo que aquí se visibiliza más que reproducir la imagen estereotipada del indígena silencioso (CASTAGNO; BRAYBOY, 2008), muestra las consecuencias de una escolarización que en medio rural y comunitario ha violentado la autoestima e identidad de los estudiantes mapuche (POBLETE, 2003; PORMA, 2015), de manera similar a lo que ha acontecido en otros contextos indígenas (BARNES; JOSEFOWITZ; COLE, 2006; COMMISION DE VÉRITÉ ET RÉCONCILIATION DU CANADA, 2015; RAU; RITCHIE, 2011). Por lo tanto, lo que tenemos es una institución donde los padres de familia no se sienten en confianza de expresar sus conocimientos e inquietudes, lo que conduce a que la comunicación con los profesores sea deficiente y limitada a temas funcionales de la escuela (TORRES, 2016). 
Lo anterior se ve profundizado por un sistema de escuelas que no está en sintonía con las características de los territorios mapuches, una condición que es problemática en la perspectiva de los actores políticos, al dificultar la superación de las incomprensiones epistémicas y ontológicas en la relación escuela-familiacomunidad. Por eso mismo basándonos en lo analizado por Garcia y Shirley (2012), se requiere de una reconceptualización de la escuela en el sentido de reposicionarla como un "lugar sagrado" (Sacred Landscapes). Es decir, un espacio en el que los profesores asumen la responsabilidad de incorporar en el trabajo en aula los conocimientos indígenas y orientan a los estudiantes indígenas en el proceso crítico de promover, proteger y preservar, sus lenguas, culturas, tierras y sociedad. Esto conlleva la ejecución de acciones que se enmarcan en un proceso político y pedagógico contra-hegemónico (BARONNET, 2017), lo que según Sartorello (2014) genera una confrontación con el modelo educativo neoliberal, al aportar nuevas formas de entender los contenidos, metodologías y formación en el medio escolar. Es decir, una educación que no siga expandiendo la competitividad, el desarraigo, la discriminación y la violencia en los estudiantes indígenas, a lo que se añade ir más allá de la ambigüedad de las respuestas y programas estatales, por representar mecanismos neocoloniales que exacerban el "mal vivir" (BERTELY; SARTORELLO; ARCOS, 2015; BERTELY, 2015). En síntesis, la educación en las escuelas en contexto mapuche debe alentar la formación de estudiantes críticos y preparados para ser conscientes de las injusticias sociales a las que se ven confrontados, $\mathrm{y}$ al mismo tiempo entregarles los conocimientos que les permitan contribuir de manera responsable a encontrar soluciones a los desafíos que enfrentan sus comunidades (GARCIA; SHIRLEY, 2012). Es este tipo de educación es la que podría aportar a la preparación de una masa crítica de estudiantes que trabajen por la construcción de la libre determinación en territorio mapuche.

\section{REFERENCIAS}

ALMONACID, Claudio. Un cuasi mercado educacional: la escuela privada subvencionada en Chile. Revista de Educación, v. 333, p. 165-196, 2004.

ASSAÉL, Jenny; ACUÑA; Felipe; CONTRERAS, Paulina; CORBALÁN, Francisca. Transformaciones en la cultura escolar en el marco de la implementación de políticas de accountability en Chile: Un estudio etnográfico en dos escuelas clasificadas en recuperación. Estudios pedagógicos, vol. 40, n. 2, p. 7-26, 2014. 
BARIBEAU, Colette. Analyse des données des entretiens de groupe. Recherches Qualitatives, v. 28, n. 1, p. 133-148, 2009.

BARNES, Rosemary; JOSEFOWITZ, Nina; COLE, Ester. Residential schools: Impact on Aboriginal students' academic and cognitive development. Canadian Journal of School Psychology, v. 21, n. 1-2, p. 18-32, 2006.

BARONNET, Bruno. Estrategias alternativas de educación en las luchas de los pueblos originarios en México. Educação \& Sociedade, v. 38, n. 140, p. 689-704, 2017.

BELLEI, Cristián. Expansión de la educación privada y mejoramiento de la educación en Chile. Evaluación a partir de la evidencia. Revista pensamiento educativo, v. 40, n. 1, p. 1-37, 2007.

BERTELY, María. Enfoques postcoloniales y movimiento político y pedagógico intercultural en y desde Chiapas, México. Relaciones. Estudios de historia y sociedad, v. 36, n. 141, p. 75-102, 2015.

BERTELY, María; SARTORELLO, Stefano; ARCOS, Francisco. Vigilancia, cuidado y control étnico-político: Red de Educación Inductiva Intercultural. Desacatos, n. 48, p. 32-49, 2015.

BOCCARA, Guillaume; BOCCARA-SEGUEL, Ingrid. Políticas indígenas en Chile (Siglos XIX y XX). De la asimilación al pluralismo (El caso mapuche). Revista de Indias, v. 59, n. 217, p. 741-774, 1999.

BOCCARA, Guillaume; AYALA, Patricia. Patrimonializar al indígena. Imaginación del multiculturalismo neoliberal en Chile. Cahiers des Amériques latines, n. 67, p. 207-228, 2011.

BOLADOS, Paola. Neoliberalismo multicultural en el Chile postdictadura: la política indígena en salud y sus efectos en comunidades mapuches y atacameñas. Chungará, v. 44, n.1, p. 135-144, 2012.

BONFIL BATALLA, Guillermo. Identidad y pluralismo cultural en América Latina. Buenos Aires: CEHASS, 1992.

BOUSQUET, Marie-Pierre. Êtres libres ou sauvages à civiliser? L'éducation des jeunes Amérindiens dans les pensionnats indiens au Québec, des années 1950 à 1970. Revue d'histoire de l'enfance «irrégulière»", Le Temps de l'histoire, n. 14, p. 162-192, 2012.

BRIONES, Claudia. Navegando creativamente los mares del disenso para hacer otros compromisos epistemológicos y ontológicos. Cuadernos de antropología social, n. 40, p. 9-70, 2014.

CALDERÓN, Margarita; CASTILLO, Silvia; FUENZALIDA, Diego; HASLER, Felipe; MARIANO, Héctor; VARGAS, Cristián. Estudio de casos de la enseñanza de la lengua y cultura mapuche y su implementación como asignatura del currículo de educación básica. Calidad en la educación, n. 47, p. 43-80, 2017. 
CANDIA DA SILVA, Fernando. La escuela misional entre los mapuche: ensayos para una «escuela indígena» en la Araucanía (1896-1924). Historia de la Educación. Anuario, v. 18, n. 2, p. 146-162, 2017.

CANO, Daniel. El rol de la mujer en la educación mapuche. En SERRANO, Sol; PONCE DE LEÓN, Macarena; RENGIFO, Francisca; MAYORGA, Rodrigo (eds.). Historia de la educación en Chile (1810-2010). Santiago: Taurus, 2018, p. 303-340.

CASTAGNO, Angelina; BRAYBOY, Bryan. Culturally responsive schooling for Indigenous youth: A review of the literature. Review of Educational Research, v. 78, n. 4, p. 941-993, 2008.

CASTILLO, Silvia; LONCON, Elisa. Noción del educador tradicional mapuche en contextos urbanos, desde la perspectiva de la dupla pedagógica. Contextos: Estudios de Humanidades y Ciencias Sociales, n. 33, p. 29-46, 2015.

COMMISSION DE VÉRITÉ ET RÉCONCILIATION DU CANADA. Honorer la vérité, réconcilier pour l'avenir: Sommaire du rapport final de la Commission de vérité et réconciliation du Canada. Montréal: McGill-Queen's University Press, 2015.

DILLEHAY, Tom; SAAVEDRA, José. Los Valles de Purén-Lumaco y Liucura, Chile: Arqueología e Historia Cultural. Nashville: Vanderbilt University, 2010.

DONOSO, Sebastián; CASTRO, Moyra; ALARCÓN, José. Aspectos críticos en las propuestas sobre una nueva arquitectura de la educación pública chilena. Estudios pedagógicos, v. 41, n. 2, p. 305-324, 2015.

ELACQUA, Gregory; SANTOS, Humberto. Preferencias reveladas de los proveedores de educación privada en Chile: el caso de la Ley de Subvención Escolar Preferencial. Gestión y política pública, v. 22, n. 1, p. 85-129, 2013.

FAVRE, Henri. L'indigénisme. Paris : Presses universitaires de France, 1996.

FLORES, Jaime; AZÓCAR, Alonso. Fotografía de capuchinos y anglicanos a principios del siglo XX: la escuela como instrumento de cristianización y chilenización. Memoria americana, n. 14, p. 75-87, 2006.

FLORES, Carolina; CARRASCO, Alejandro. Elegir lo que hay: ¿Cuentan las familias en sus barrios con una oferta de escuelas que respondan a sus preferencias? En CORVALÁN, Javier; CARRASCO, Alejandro; GARCÍA-HUIDOBRO, Juan (eds). Mercado escolar: Libertad, diversidad y desigualdad. Santiago: Ediciones Universidad Católica de Chile, 2016, p. 151-187.

FUENZALIDA, Pedro. Re-etnización y descolonización: resistencias epistémicas en el currículum intercultural en la Región de Los Lagos-Chile. Polis. Revista Latinoamericana, n. 38, 2014.

GARCIA, Jeremy; SHIRLEY, Valerie. Performing decolonization: Lessons learned from Indigenous youth, teachers and leaders' engagement with critical Indigenous pedagogy. Journal of Curriculum Theorizing, v. 28, n. 2, p. 76-91, 2012. 
GOBIERNO DE CHILE. Informe de la Comisión Verdad Histórica y Nuevo Trato. Informes Finales de los Grupos de Trabajo. v. 3. Anexo Tomo II, 2003. http://www.memoriachilena. gob.cl/602/articles-122901_recurso_7.pdf. Acceso en: mar. 2020.

GONZÁLEZ APODACA, Erica. Apropiación étnica de la escuela entre los pueblos mixes de Oaxaca: La educación comunitaria e intercultural vista desde sus jóvenes. Perfiles educativos, v. 35, n. 141, p. 65-83, 2013.

LAGOS, Cristián. El Programa de Educación Intercultural Bilingüe y sus resultados: ¿perpetuando la discriminación? Pensamiento Educativo. Revista de Investigación Educacional Latinoamericana, v. 52, n. 1, p. 84-94, 2015.

L'ÉCUYER, René. Méthodologie de l'analyse développementale de contenu: Méthode GPS et concept de soi. Québec: Les Presses de l’Université du Québec, 1990.

LOPES DA SILVA, Silvia. Indigenous School Policies and Politics: The Sociopolitical Relationship of Wayãpi Amerindians to Brazilian and French Guianan Schooling. Anthropology \& Education Quarterly, v. 40, n. 2, p. 170-186, 2009.

LOPES, Silvia. Nous allons à l'école pour en savoir plus!» Un regard sur les projets éducatifs des Wayãpi en Amazonie brésilienne. En BELLIER, Irène; HAYS, Jennifer (eds.). Quelle éducation pour les peuples autochtones? Paris: L'Harmattan, 2016, p. 147-161.

LÓPEZ, Luis. L'éducation des populations autochtones et les droits des peuples autochtones en Amérique latine. En BELLIER, Irène; HAYS, Jennifer (eds.). Quelle éducation pour les peuples autochtones? Paris: L'Harmattan, 2016, p. 37-58.

LUNA, Laura; BOLOMEY, Carlos; CANIGUAN, Natalia. Educación mapuche en el Chile neoliberal: análisis de tres escuelas de la región de La Araucanía. Sinéctica, n. 50, 2018.

LUNA, Laura; BENAVIDES, Pelayio; GUTIÉRREZ, Pablo; ALCHAO, Manuela; DITTBORN, Alberto. Aprender lengua y cultura mapuche en la escuela: estudio de caso de la implementación del nuevo Sector de Aprendizaje Lengua Indígena desde un análisis de recursos educativos. Estudios Pedagógicos, v. 40, n. 2, p. 221-240, 2014.

MANSILLA, Juan; HUAIQUIAN, Claudia; POZO, Gabriel. Infancia mapuche encerrada: internados de las escuelas-misiones en la Araucanía, Chile (1900-1935). Revista Brasileira de Educação, v. 23, p. 1900-1935, 2018.

MANSILLA, Juan; LLANCAVIL, Daniel; MIERES, Manuel; MONTANARES, Elizabeth. Instalación de la escuela monocultural en la Araucanía, 1883-1910: dispositivos de poder y Sociedad Mapuche. Educação e Pesquisa, v. 42, n. 1, p. 213-228, 2016.

MCCARTY, Teresa. A place to be Navajo: Rough Rock and the struggle for selfdetermination in indigenous schooling. Estados Unidos: Routledge, 2010.

MCGREGOR, Heather. Curriculum change in Nunavut: Towards Inuit Qaujimajatuqangit. McGill Journal of Education/Revue des sciences de l'éducation de McGill, v. 47, n. 3, p. 285-302, 2012. 
MILES, Matthew B.; HUBERMAN, Michael A. Analyse des données qualitatives. Bruxelles: De Boeck Supérieur, 2003.

MINISTERIO de EDUCACIÓN. Orientaciones para la contextualización de Planes y Programas para la Educación Intercultural Bilingüe. Santiago: Gobierno de Chile, 2005a.

MINISTERIO de EDUCACIÓN. Manual instructivo para la implementación del PEI intercultural. Santiago: Gobierno de Chile, $2005 \mathrm{~b}$.

MINISTERIO de EDUCACIÓN. Programa de Educación Intercultural Bilingüe (2010-2016). Santiago: Ministerio de Educación, 2017.

MIZALA, Alejandra; ROMAGUERA, Pilar. Evaluación del desempeño e incentivos en la educación chilena. Cuadernos de economía, v. 39, n. 118, p. 353-394, 2002.

NIGH, Ronald; BERTELY, Maria. Conocimiento y educación indígena en Chiapas, México: un método intercultural. Diálogos sobre educación. Temas actuales en investigación educativa, v. 9, n. 16, 2018.

NUÑEZ, Carmen G.; SOLÍS, Camila; SOTO, Rodrigo. ¿Qué sucede en las comunidades cuando se cierra la escuela rural? Un análisis psicosocial de la política de cierre de las escuelas rurales en Chile. Universitas Psychologica, v. 13, n. 2, p. 615-625, 2014.

PAQUETTE, Jerard; FALLON, Gerald. Significado del control aborigen de la educación bajo los actuales acuerdos de autogobierno en Canadá. Revista Educación y Pedagogía, v. 22, n. 58, p. 193-212, 2010.

PARCERISA, Lluís; FALABELLA, Alejandra. La consolidación del Estado evaluador a través de políticas de rendición de cuentas: trayectoria, producción y tensiones en el sistema educativo chileno. Education Policy Analysis Archives/Archivos Analíticos de Politicas Educativas, n. 25, p. 1-24, 2017.

PINTO, Jorge. De la inclusión a la exclusión. La formación del estado, la nación y el pueblo mapuche. Santiago: Universidad de Santiago, 2003.

POBLETE, Maíra Pía. Discriminación étnica en relatos de la experiencia escolar mapuche en Panguipulli (Chile). Estudios pedagógicos, n. 29, p. 55-64, 2003.

PORMA, Juan. Violencia colonial en la Escuela: el caso de la comunidad José Porma en el siglo XX. En ANTILEO, Enrique; CÁRCAMO-HUECHANTE, Luis; CALFÍO, Margarita; HUINCA-PITRÚN, Herson (eds.). Awükan ka kuxankan zugu wajmapu mew. Santiago: Ediciones Comunidad de Historia Mapuche, 2015, p. 189-205.

POZO, Gabriel. ¿Cómo descolonizar el saber? El problema del concepto de interculturalidad. Reflexiones para el caso mapuche. Polis. Revista Latinoamericana, n. $38,2014$.

QUIDEL, Gloria. Estrategias de enseñanza de la lengua mapunzugun en el marco del PEIB Mineduc-Orígenes (Comuna de Padre las Casas, IX Región-Chile). Cuadernos Interculturales, v. 9, n. 16, 2011. 
QUILAQUEO, Daniel; SARTORELLO, Stefano. Retos epistemológicos de la interculturalidad en contexto indígena. Alpha (Osorno), n. 47, p. 47-61, 2018.

QUIVY, Rayrnond; CAMPENHOUDT, Luc Van. Manual de investigación en ciencias sociales. México: Limusa, 1998.

RAU, Cheryl; RITCHIE, Jenny. Ahakoa he iti: Early childhood pedagogies affirming of Māori children's rights to their culture. Early Education \& Development, v. 22, n. 5, p. 795-817, 2011.

RESCHIO, Antonio. La Araucanía memorias inéditas de la Misión capuchina en Chile (1848-1890). Temuco: Ofqui Editores, 2018.

RICO, Braden. Awakening vision: examining the reconceptualization of Aboriginal education in Canada via Kaupapa Māori praxis. Asia Pacific Journal of Education, v. 33, n. 4, p. 380-393, 2013.

ROCKWELL, Elsie. La escuela cotidiana. México: Fondo de Cultura Económica, 1997.

ROCKWELL, Elsie. La experiencia etnográfica: historia y cultura en los procesos educativos. Buenos Aires: Paidós, 2009.

SALAÜN, Marie; VERNAUDON, Jacques. La citoyenneté comme horizon: destin commun, demande sociale et décolonisation de l'école en Nouvelle-Calédonie aujourd'hui. Anthropologie et sociétés, v. 33, n. 2, p. 63-80, 2009.

SÁNCHEZ, Raúl. La empresa económica jesuita en el obispado de Concepción: el caso de los colegios San Bartolomé de Chillán y Buena Esperanza. Universum, v. 26, n. 2, p. 215-243, 2011.

SARTORELLO, Stefano. La co-teorización intercultural de un modelo curricular en Chiapas, México. Revista mexicana de investigación educativa, v. 19, n. 60, p. 73-101, 2014.

SERRANO, Sol; PONCE DE LEÓN, Macarena; RENGIFO, Fracisco. Historia de la educación en Chile (1810-2010). Tomo II La educación nacional (1880-1930). Santiago: Taurus, 2013.

SIMPSON, Leanne. Land as pedagogy: Nishnaabeg intelligence and rebellious transformation. Decolonization: Indigeneity, Education \& Society, v. 3, n. 3, 2014.

SLACHEVSKY, Natalia. Una revolución neoliberal: la política educacional en Chile desde la dictadura militar. Educação e Pesquisa, v. 41, 1473-1486, 2015.

SOTOMAYOR, Carmem; ALLENDE, Claudia; FUENZALIDA, Diego; HASLER, Felipe; CASTILLO, Silvia. Competencias lingüísticas e interculturales de los educadores tradicionales mapuche para la implementación de la asignatura de Lengua Indígena en Chile. Santiago: CIAE, Universidad de Chile, 2015.

TOCKER, Kimai. The origins of kura kaupapa Māori. New Zealand Journal of Educational Studies, v. 50, n. 1, p. 23-38, 2015. 
TORRES, Héctor. La educación intercultural bilingüe en Chile: Experiencias cotidianas en las escuelas de la región mapuche de La Araucanía. Québec, Université Laval, 2017.

TORRES, Héctor. La escuela y la puesta en práctica de la EIB en la perspectiva de padres en medio mapuche. EDUCADI, v. 1, n. 2, 2016.

TUBINO, Fidel. Interculturalidad para todos: ¿un slogan más? PALESTRA, Pontificia Universidad Católica del Perú, 2011.

VALENZUELA, Juan; BELLEI, Cristián; DE LOS RÍOS, Danae. Segregación escolar en Chile. En MARTINIC, Sergio; ELACQUA, Gregory (eds.). Cambios en la gobernanza del sistema educativo chileno. Santiago: UNESCO, Pontificia Universidad Católica de Chile, 2010, p. 257-284.

VILLALOBOS, Cristobal; QUARESMA, Maria. Sistema escolar chileno: características y consecuencias de un modelo orientado al mercado. Convergencia Revista de Ciencias Sociales, v. 22, n. 69, p. 63-84, 2015.

VILLALOBOS, Cristobal; VALENZUELA, Juan. Polarización y cohesión social del sistema escolar chileno. Revista de análisis económico, v. 27, n. 2, p. 145-172, 2012.

WEENIE, Angelina. Curricular theorizing from the periphery. Curriculum Inquiry, v. 38, n. 5, p. 545-557, 2008.

WEINSTEIN, José; MUÑOZ, Gonzalo; MARFÁN, Javiera. Liderar bajo presión: las estrategias gestionadas por los directores de escuela para alcanzar los resultados comprometidos. En WEINSTEIN, José; MUÑOZ, Gonzalo (eds.). ¿Qué sabemos sobre los directores de escuela en Chile? Santiago: Fundación Chile, 2012, p. 219-248.

WILLIAMSON, Guillermo; FLORES, Fabián. Estado del arte de la Educación Intercultural bilingüe en Chile, 1990-2013. Temuco: ICIIS y Ediciones Universidad de La Frontera, 2015.

Texto recibido el 18/04/2019.

Texto aprobado el 31/03/2020. 